\title{
Capacitive Sensor Circuit with Relative Slope-Boost Method Based on a Relaxation Oscillator
}

\author{
Ryo Onishi Koki Miyamoto Korkut Kaan Tokgoz Noboru Ishihara Hiroyuki Ito \\ Nano Sensing Unit, Institute of Innovative Research, Tokyo Institute of Technology \\ 4259- J2-31, Nagatsuta-cho, Midori-ku, Yokohama, Kanagawa, 226-8503, Japan \\ E-mail: paper@1si.pi.titech.ac.jp
}

\begin{abstract}
This paper presents a relative slope-boosting technique for a capacitive sensor circuit based on a relaxation oscillator. Our technique improves jitter, i.e. resolution, by changing both the voltage slope on the sensing and the reference sides with respect to the sensor capacitance. The sensor prototype circuit is implemented in a 180-nm standard CMOS process and achieves resolution of $710 \mathrm{aF}$ while consuming $12.7 \mathrm{pJ}$ energy every cycle of $13.78 \mathrm{kHz}$ output frequency. The measured power consumption from a 1.2 V DC supply is $430 \mathrm{nW}$.
\end{abstract}

\section{KEYWORDS}

Capacitive sensor circuit, CMOS, jitter, relaxation oscillator, slopeboost, ultra-low power

\section{Introduction}

Capacitive sensor circuits can be used for many applications, such as sensing acceleration, pressure and so on [1-3]. The type of capacitive sensors converting the capacitance value to period is called Period Modulation (PM) type. Fig. 1 (a) shows the schematic of the general PM-type capacitive sensor circuit. The PM-type sensor circuit has mainly composed of a relaxation oscillator, generating square wave with periods proportional to the values of resistance and capacitance [4]. As the period of the square wave is counted, the value of capacitance is converted to digital value easily.

The challenge of the PM-type sensor circuits compared to other sensor circuits is low resolution which depends on jitter generated at the relaxation oscillator. Fig. 1 (b) illustrates the process of jitter generation, where $V_{\text {noise }}$ is input referred noise of the comparator in the relaxation oscillator. The voltage of minus input terminal at the comparator is $R I+V_{\text {noise. Thus, }} V_{\text {noise }}$ disturbs the timing of logic inversion at reset logic connected at the output of the comparator, and this disturbed timing is called jitter which depends on the voltage slope in this circuit topology [5]. There is a trade-off between the voltage slope and current consumption. Therefore, we need to decrease the jitter to achieve finer resolution while care should be given on keeping the energy consumption low.

Permission to make digital or hard copies of all or part of this work for personal or classroom use is granted without fee provided that copies are not made or distributed for profit or commercial advantage and that copies bear this notice and the full citation on the first page. Copyrights for components of this work owned by others than the author(s) must be honored. Abstracting with credit is permitted. To copy otherwise, or republish, to post on servers or to redistribute to lists, requires prior specific permission and/or a fee. Request permissions from Permissions@acm.org. ASPDAC '21, January 18-21, 2021, Tokyo, Japan

(C) 2021 Copyright is held by the owner/author(s). Publication rights licensed to ACM.

ACM ISBN 978-1-4503-7999-1/21/01 ..\$15.00

https://doi.org/10.1145/3394885.3431655
This work proposes jitter reduction technique increasing the voltage slope of input terminal at the comparator, which is explained in the next section. Section 3 focuses on the measurement results. Finally, conclusions are provided in Section 4.

\section{Slope-Boost Capacitive Sensing Circuit}

Fig. 2 explains the proposed relative slope-boost technique. By comparing $V_{\mathrm{C}}$ and reversed slope $V_{\text {Ref, the voltage slope relatively }}$ increases to Slope - -Slope Ref, and jitter can be reduced by $33 \%$ in simulation with very small increase in energy consumption.

Fig. 3 illustrates the schematic of the proposed capacitive sensor circuit composed of a differential comparator, a latch, a subtraction circuitry, resistance, and capacitance. When $\Phi$ is high, the input voltage at comparator is

$$
\begin{aligned}
& V_{\mathrm{C}}=V_{\mathrm{dd}} \cdot\left\{1-\exp \left(-\frac{1}{R C_{\mathrm{m}}} \mathrm{t}\right)\right\} \\
& V_{\mathrm{R}}=V_{\mathrm{dd}}-V_{\mathrm{C}}=V_{\mathrm{dd}} \cdot \exp \left(-\frac{1}{R C_{\mathrm{m}}} \mathrm{t}\right),
\end{aligned}
$$

where $C_{\mathrm{m}}$ is capacitance of the sensor. When $V_{\mathrm{R}}$ matches $V_{\mathrm{C}}$, comparator output reverses. The measurement time can be calculated as $R C_{\mathrm{m}} \ln (2)$. When $\Phi$ is low, reference time can be calculated as $R C_{\text {ref }} \ln (2)$ similarly, where $C_{\text {ref }}$ is reference capacitance. Therefore, oscillation period is $R\left(C_{\mathrm{m}}+C_{\mathrm{ref}}\right) \ln (2)$, and $C_{\mathrm{m}}$ can be measured from oscillation period as $R$ and $C_{\text {ref }}$ is known.

\section{Measurement Result}

Fig. 4 shows the photograph of a test board and a prototype circuit IC fabricated in a $180-\mathrm{nm}$ standard CMOS process. The core area of our circuit is $198-\mu \mathrm{m} \times 280-\mu \mathrm{m}$. Fig. 5 shows capacitance vs. period characteristic showing linear behavior. Fig. 6 provides the measurement result at $C_{\mathrm{m}}=5 \mathrm{pF}$. The measured output frequency is $16.8 \mathrm{kHz}$, and resolution is $710 \mathrm{aF}$ while consuming $25.7 \mathrm{pJ}$ every cycle from a $1.2 \mathrm{~V}$ power supply. Measured jitter is $4.92 \mathrm{~ns}$, and current and power consumptions are $359 \mathrm{nA}$ and $430 \mathrm{nW}$. This jitter can be reduced by time averaging. The red dots in Fig. 6 are plotted with number of averaging period ( $N$ period) of $0,2,5,10,20,50$, $100,200,500,1000,2000,5000$, and 10000; from left to right. The best resolution is obtained at $710 \mathrm{aF}$ when $N$ is 50 ,

\section{Conclusions}

This paper proposes relative slope-boost technique for reducing the jitter caused by the comparator in the PM-type capacitive sensor circuit. The proposed capacitive sensor circuit achieves low energy consumption and high resolution compared to state-of-the-art capacitive sensor circuits as shown in Table I. As a result, the proposed circuit technique can contribute to longer battery life of IoT devices, with accurate sensor readings. 


\section{ACKNOWLEDGMENTS}

This work was partly supported by KAKENHI (JP17H01279 JP19H02191), and VDEC in collaboration with Cadence Design Systems, Inc., Keysight Technologies Japan, Ltd., and Mentor Graphics, Inc.

\section{REFERENCES}

[1] A. Alhoshany and K. N. Salama, "A Precision, Energy-Efficient, Oversampling, Noise-Shaping Differential SAR Capacitance-to-Digital Converter," IEEE Trans. Instrum. Meas., vol. 68, no. 2, pp. 392-401, 2019.

[2] Y. Luo and C. Heng, "Capacitance-to-Digital Converter with Reduced Data Throughput," Symp. VLSI Circuits, pp. 165-166, 2018.

[3] Y. He, Z.-Y. Chang, L. Pakula, S. H. Shalmany, and M. Pertijs, "A 0.05 $\mathrm{mm}^{2} 1 \mathrm{~V}$ capacitance-to-digital converter based on period modulation," IEEE ISSCC Dig.Tech. Papers, pp. 486-487, 2015.

[4] D. Griffith, P. T. Roine, J. Murdock, and R. Smith, “A 190nW 33kHz RC Oscillator with $\pm 0.21 \%$ Temperature Stability and 4ppm Long-Term Stability," ISSCC Dig.Tech. Papers, pp. 300-301, 2014.

[5] J. Lee, A. George, and M. Je, "A 1.4V 10.5 MHz swing-boosted differential relaxation oscillator with $162.1 \mathrm{dBc} / \mathrm{Hz}$ FOM and 9.86psrms period

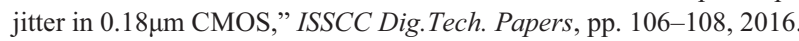

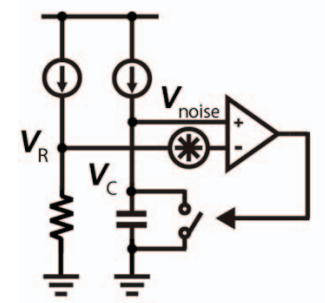

(a)

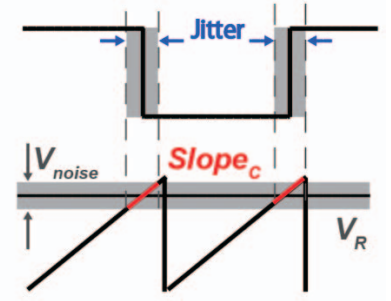

(b)
Figure 1: (a) Conventional PM-type capacitive sensor circuit based on relaxation oscillator, and (b) output voltage waveform illustrating the cause of jitter.

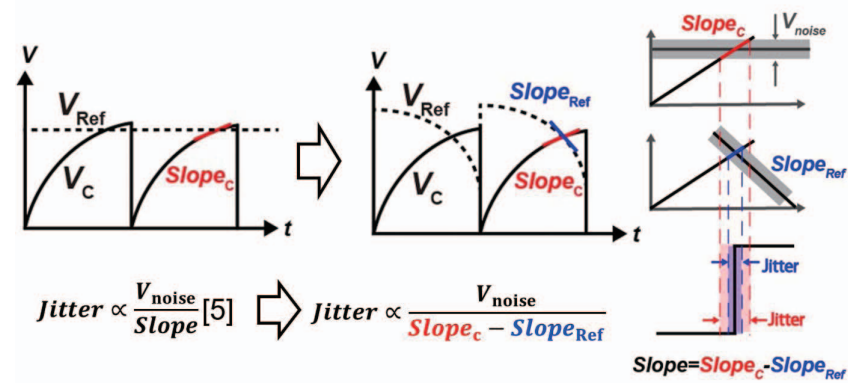

Figure 2: The proposed relative slope-boost to reduce jitter.

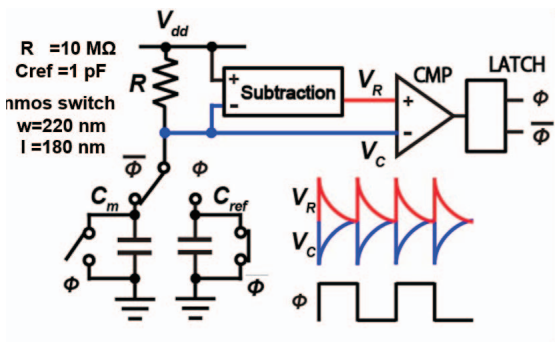

(a)

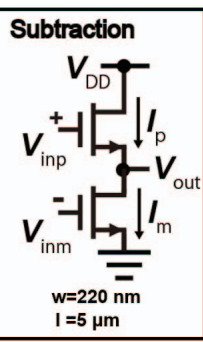

(b)
Figure 3: (a) The proposed capacitive sensor circuit based on relaxation oscillator, (b) schematic of the subtraction circuit for reversed slope generation and these parameters.

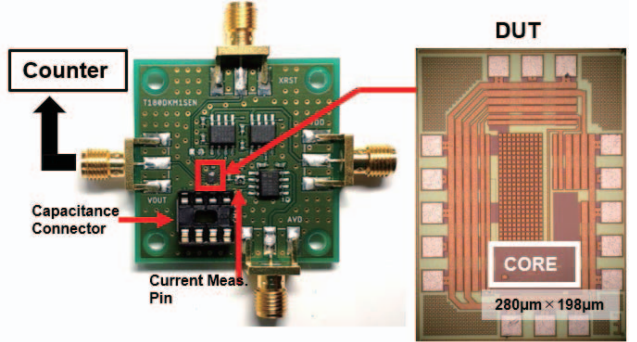

Figure 4: Test board and the prototype circuit in $180 \mathrm{~nm}$ CMOS.

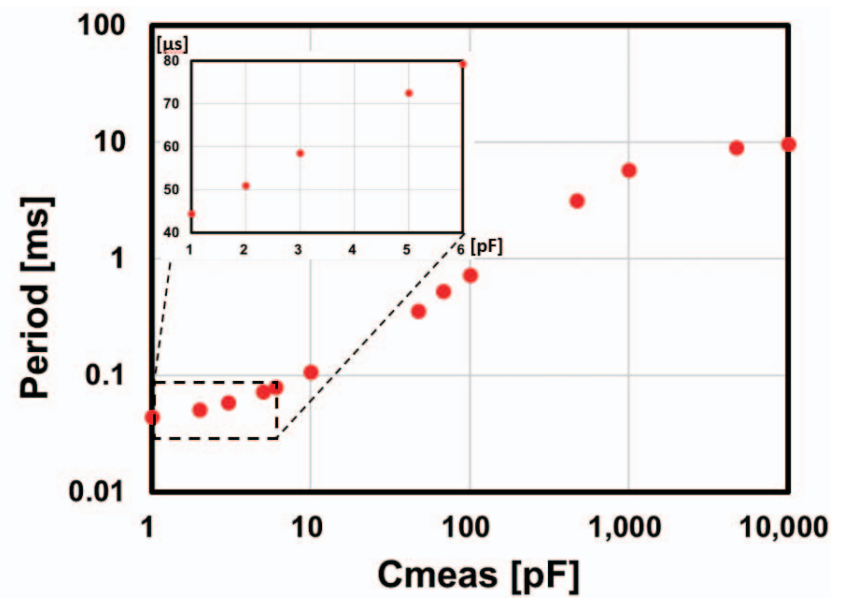

Figure 5: Measured period vs. measured capacitance

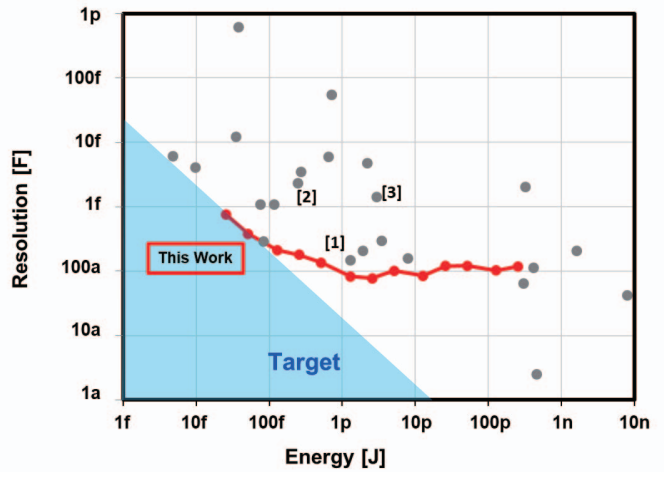

Figure 6: Benchmark

Table 1: Comparison

\begin{tabular}{|c|c|c|c|c|}
\hline & $\begin{array}{c}\text { TRANS } 2019 \\
\text { [1] }\end{array}$ & $\begin{array}{c}\text { VLSI } 2018 \\
{[2]}\end{array}$ & $\begin{array}{c}\text { ISSCC } 2015 \\
{[3]}\end{array}$ & This Work \\
\hline $\begin{array}{c}\text { Tech } \\
\text { [nm CMOS] }\end{array}$ & 180 & 130 & 160 & 180 \\
\hline Type & SAR & PM & PM & PM \\
\hline Power $[\mu \mathrm{W}]$ & 1.59 & 8.2 & 14 & $0.43^{*}$ \\
\hline $\begin{array}{l}\text { Input range } \\
{[\mathrm{pF}]}\end{array}$ & 3.6 & 20 & $0-8$ & $0-197$ \\
\hline $\begin{array}{l}\text { MeasTime } \\
\quad[\mu \mathrm{s}]\end{array}$ & 810 & 482 & 210 & $59.7^{*}$ \\
\hline Energy[fJ] & 1290 & 116 & 2940 & 25.7 \\
\hline $\begin{array}{c}\text { Resolution } \\
{[\mathrm{aF}]}\end{array}$ & 150 & 2345 & 1443 & $710^{*}$ \\
\hline
\end{tabular}

* measurement with $5 \mathrm{pF}$ 